\title{
Effect of three prophylaxis methods on surface roughness of giomer
}

\author{
Soodabeh Kimyai ${ }^{1}$, Siavash Savadi-Oskoee ${ }^{1}$, Amir-Ahmad Ajami ${ }^{2}$, Alireza Sadr ${ }^{3}$, Saeedeh Asdagh ${ }^{4}$
}

\author{
${ }^{1}$ DDS, MS: Associate Professor. Department of Operative Dentistry, School of Dentistry, Tabriz University of Medical Sci- \\ ences, Tabriz, Iran \\ ${ }^{2}$ DDS, MS: Assistant Professor. Department of Operative Dentistry, School of Dentistry, Tabriz University of Medical Sci- \\ ences, Tabriz, Iran \\ ${ }^{3}$ DDS, Ph.D: Assistant Professor and Principal Investigator. Department of Cariology and Operative Dentistry, Global Center \\ of Excellence, IRCMSTBD, Tokyo Medical and Dental University, Tokyo, Japan \\ ${ }^{4}$ DDS: Post graduate student. Department of Operative Dentistry, School of Dentistry, Tabriz University of Medical Sciences, \\ Tabriz, Iran
}

\author{
Correspondence: \\ Department of Operative Dentistry, \\ Tabriz University of Medical Sciences, \\ School of Dentistry, \\ Gholghasht Street, Zip: 5166614713, \\ Tabriz, Iran \\ soodabehkimyai@yahoo.com
}

Received: 07-02-2010

Accepted: $31-05-2010$

\author{
Kimyai S, Savadi-Oskoee S, Ajami AA, Sadr A, Asdagh S. Effect of three \\ prophylaxis methods on surface roughness of giomer. Med Oral Patol \\ Oral Cir Bucal. 2011 Jan 1;16 (1):e110-4. \\ http://www.medicinaoral.com/medoralfree01/v16i1/medoralv16ilp110.pdf

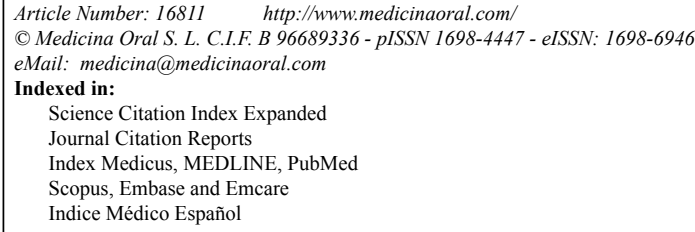

\begin{abstract}
Objectives: Plaque and stains are removed by prophylaxis methods from tooth surfaces. Since prophylaxis methods can have a detrimental effect on the surface finish of restorations, the aim of this in vitro study was to investigate the effect of three prophylaxis methods, including pumice with rubber cup, pumice with brush, and air-powder polishing device (APD) on the surface roughness of giomer.

Study design: Sixty four cylindrical giomer (Beautifil II, Shofu) samples with a diameter of $6 \mathrm{~mm}$ and a height of $2 \mathrm{~mm}$ were used. Subsequent to a 3 -month period of storage in distilled water at $37^{\circ} \mathrm{C}$, the samples were randomly divided into four groups of 16. In group 1 (control), no prophylaxis procedure was carried out. In groups 2 to 4 the samples were exposed to pumice with rubber cup, pumice with brush, and APD prophylaxis methods, respectively. The surface roughness of the samples was measured using a profilometer and the effect of different prophylaxis methods on surface topography was characterized by atomic force microscopy (AFM). All data were analyzed by one-way ANOVA and Duncan's post hoc test at a significance level of $\mathrm{P}<0.05$.

Results: There were statistically significant differences in surface roughness among the groups $(\mathrm{P}<0.0005)$. Furthermore, in pairwise comparisons there were statistically significant differences between all the groups $(\mathrm{P}<$ 0.05). The roughest surfaces, in descending order, were observed with the use of APD, pumice with brush, and pumice with rubber cup.

Conclusions: The use of different prophylaxis methods resulted in an increased surface roughness of giomer compared with the control group. APD prophylaxis exerted the most detrimental effects on the surface of giomer.
\end{abstract}

Key words: Giomer, prophylaxis methods, surface roughness. 


\section{Introduction}

The dental plaque is considered a complex bacterial ecosystem that undergoes evolution, maturation and development, leading to odontogenic infections (1). Prophylaxis methods are used to mechanically remove plaque and stain from tooth surfaces; such methods give rise to loss of superficial structure and roughen surfaces as a result of their abrasive action, depending on the method used and the time spent $(2,3)$. It has been reported that prophylaxis methods have a detrimental effect on the surface finish of restorations $(3,4)$. Since stains and plaque deposit more in the cervical area of teeth near the gingival tissue, cervical restorations are the most influenced ones by prophylaxis methods (3). Rough restoration surfaces favor staining, plaque retention and gingival irritation $(4,5)$.

One of the most common prophylaxis procedures is the use of pumice along with carriers, such as rotating rubber cups or brushes $(2,3)$. The air-powder polishing device (APD) is the alternative method and it has been reported that the use of APD is more efficacious and time-saving compared to rubber cup with pumice (6-8). However, APD is more aggressive and dentin structure loss might occur when this method is used (2). With APD, sodium bicarbonate particles are propelled by air jet to combine with a small stream of water; as a result, slurry is created, which is directed onto the tooth surface (4).

In previous studies influence of different prophylaxis procedures on surface roughness of different types of composite resins and glass-ionomers has been investigated and it has been reported that the effect of prophylaxis treatments depends on the material $(3,4)$. Recently a new group of hybrid aesthetic restorative materials known as giomers has been introduced. Giomers can be acceptable restorative materials for cervical restorations. They use pre-reacted glass-ionomer (PRG) technology to produce a stable phase of glass-ionomer in the restorative material. The fluoroalumina silicate glass reacts with polyalkenoic acid in water before being incorporated into the silica-filled urethane resin (9-11). These materials have the fluoride release and recharge properties of glass-ionomer cements; other advantages include excellent aesthetics, easy polishability and strength of the resin composites (10). Long-term clinical studies have reported satisfactory visual texture and surface roughness of giomer restorations $(12,13)$. There is little information available about the influence of prophylaxis procedures on giomers. The aim of the present study was to evaluate the effect of three prophylaxis methods, including pumice with rubber cup, pumice with brush, and APD on giomer surface roughness.

\section{Materials and Methods}

A giomer restorative material (A3 shade, Beautifil II, Shofu Dental Corporation, Osaka, Japan) was investigated in this in vitro study.

\section{Sample preparation}

Sixty four cylindrical giomer samples with a diameter of $6 \mathrm{~mm}$ and a height of $2 \mathrm{~mm}$ were used. Giomer was placed in a Teflon mold (with an internal diameter of $6 \mathrm{~mm}$ and a height of $2 \mathrm{~mm}$ ) and covered with matrix strips (Hawe Neos Dental, Bioggio, Switzerland). A glass slide was placed over this and pressure was applied to extrude excess material. Then giomer samples were light-cured for 40 seconds through the glass slide with a conventional quartz halogen light-curing unit (Astralis 7, Ivoclar Vivadent, FL-9494 Schaan, Liechtenstein). An 8-mm-diameter probe was placed perpendicular to the surface of the samples for curing. Subsequent to discarding the matrix strips and mold removal, the samples were light-cured for another 80 seconds (20 seconds from each side). The samples were stored in distilled water at $37^{\circ} \mathrm{C}$ for three months to simulate the recall period for maintenance therapy in clinical settings (3). Then they were randomly divided into four groups of 16. In group 1 no prophylaxis procedure was carried out. In group 2 the surface of the samples was subjected to pumice-water slurry (Kemdent, Swindon, Wiltshire, UK) with a rotating rubber cup (Stoddard, Letchworth, Hertfordshire, UK) for 12 seconds in a contra-angle slow-speed handpiece at $2000 \mathrm{rpm}$. Prophylaxis agent was replaced every 6 seconds and a new rubber cup was used for each sample. In group 3 the same procedure, as described for group 2, was carried out except for the fact that instead of a rotating rubber cup, a rotating brush (Vericom Dental, Anyang, Gyeonggi, South Korea) was used. The surfaces of the samples in group 4 were treated using an air-powder polishing device (AirFlow, Electronic Medical Systems, Nyon, Switzerland). The jet tip was aligned perpendicular to the target area at a distance of $10 \mathrm{~mm}$. According to a previously described method $(3,4)$, during the first 6 seconds regular paste was used; then it was replaced with a fine paste for the next 6 seconds of the treatment. In groups 2-4, subsequent to the prophylaxis procedures the samples were rinsed in running water and further cleaned in an ultrasonic bath for 10 minutes (14).

Surface roughness measurement

The average surface roughness ( $\mathrm{Ra}, \mu \mathrm{m})$ of each sample was measured by a profilometer (MarSurf PS 1, Mahr $\mathrm{GmbH}$, Esslingen, Germany). Three readings were taken from each sample. Each giomer sample was rotated $120^{\circ}$ to record three readings per surface.

Surface topography characterization

The surface topography of giomer in the control group and groups 2-4, subsequent to the use of different prophylaxis methods, was characterized by an atomic force microscope [AFM] (NanoScope II, Digital Instruments, Santa Barbara, CA, USA). Two additional specimens in each group were prepared for AFM characterization. Sample preparation was the same as described for pro- 
filometry. In groups 2-4, subsequent to the prophylaxis procedures the samples were rinsed in running water and further cleaned in an ultrasonic bath for 10 minutes. AFM was used in contact mode using silicon nitride tip (with nominal radius of $50 \mathrm{~nm}$ and apex angle of $45^{\circ}$ ), which was connected to a fixed substrate on a cantilever. The images were recorded with a scan rate of 1.9 $\mathrm{Hz}$ and a resolution of $256 \times 256$ pixels per image. For each specimen, two scans were carried out at each specimen surface quadrant at a scanning area of $10 \mu \mathrm{m} \times 10$ $\mu \mathrm{m}$. The collected 3D topographical data was analyzed with a data analysis software (NanoScope III, Version 5.12r2, Digital Instruments, Santa Barbara, CA, USA). For each group, the surface roughness was defined as the root mean square (rms) value of surface departures within the sampling area, calculated in $\mathrm{nm}$. Moreover, the surface area difference was calculated in percent [\%] (the difference between surface area following different treatments and the smoothest condition).

Statistical analysis

All the data for surface roughness and surface area difference were analyzed with one-way ANOVA and pairwise comparisons were performed by Duncan's post hoc test. In this study statistical significance was set at $\mathrm{P}<0.05$.

\section{Results}

\section{Surface roughness}

Surface roughness ( $\mathrm{Ra}$ means and standard deviations) for the groups are shown in Table 1. There were statistically significant differences in surface roughness among the groups $\left(\mathrm{F}_{3,60}=155.92, \mathrm{P}<0.0005\right)$. Duncan pairwise comparisons showed that there were significant differences in surface roughness between all the groups $(\mathrm{P}<0.05)$.

\section{Surface topography characterization}

Selected AFM images of the surfaces of the non-treated group and specimens with prophylaxis treatments are presented in (Fig. 1. A-D). AFM images show a relatively smooth natural surface in Group 1 (Fig. 1A). In-
Table 1. Means of Ra values $(\mu \mathrm{m})$ and standard deviations (SD) for study groups; values followed by different letters were significantly different by Duncan test.

\begin{tabular}{|c|c|c|}
\hline Groups & Prophylaxis method & Mean \pm SD \\
\hline 1 & None & $0.05 \pm 0.02^{\mathrm{a}}$ \\
\hline 2 & Pumice with rubber cup & $0.59 \pm 0.21^{\mathrm{b}}$ \\
\hline 3 & Pumice with brush & $1.90 \pm 0.50^{\mathrm{c}}$ \\
\hline 4 & APD & $2.35 \pm 0.42^{\mathrm{d}}$ \\
\hline
\end{tabular}

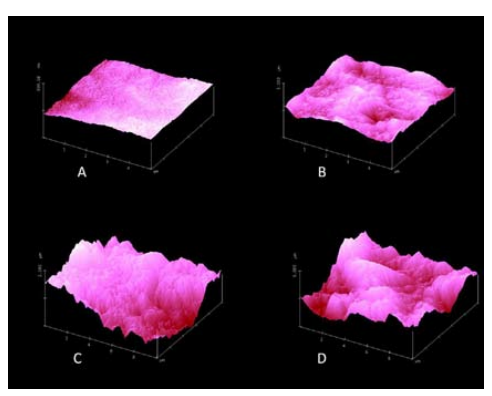

Fig. 1. AFM 3D topographical images of the surfaces of non-treated specimens (A) and specimens with pumice with rubber cup (B), pumice with brush (C) and APD (D) prophylaxis treatments.

creased surface irregularities are observed in the AFM images of other groups (Fig. 1B-D).

The results of statistical analysis of the surface roughness (rms) and surface area difference (\%) from the AFM images are presented in Table 2.

There were statistically significant differences in surface roughness and surface area difference among the groups $(\mathrm{P}<0.0005)$. Moreover, according to Duncan test the differences in surface roughness and surface area difference between all the groups were statistically significant $(\mathrm{P}<0.05)$.

Table 2. Results of statistical analysis of the surface roughness [Rms ( $\mathrm{nm})]$ and surface area difference [\%] obtained from the AFM images; mean values with dissimilar letters in the same column are statistically significant at $\mathrm{P}<0.05$.

\begin{tabular}{|c|c|c|c|}
\hline Groups & Prophylaxis method & Rms (nm), Mean \pm SD & Surface area difference (\%), Mean \pm SD \\
\hline 1 & None & $15.36 \pm 1.97^{\mathrm{a}}$ & $1.06 \pm 0.34^{\mathrm{a}}$ \\
\hline 2 & Pumice with rubber cup & $135.72 \pm 8.33^{\mathrm{b}}$ & $16.03 \pm 2.41^{\mathrm{b}}$ \\
\hline 3 & Pumice with brush & $221.15 \pm 5.47^{\mathrm{c}}$ & $24.20 \pm 2.09^{\mathrm{c}}$ \\
\hline 4 & APD & $330.82 \pm 7.09^{\mathrm{d}}$ & $31.47 \pm 0.42^{\mathrm{d}}$ \\
\hline
\end{tabular}




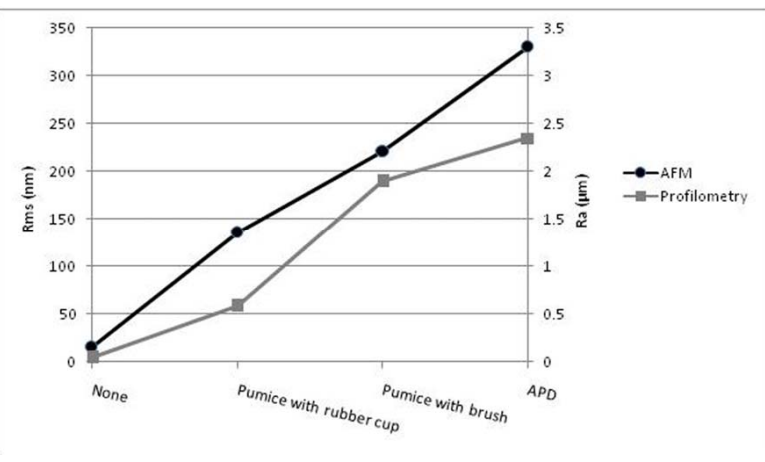

Fig. 2. The graph of surface roughness values obtained from profilometry and AFM in study groups.

The graph of surface roughness values obtained from profilometry and AFM in study groups is shown in Fig. 2.

\section{Discussion}

Surface finish quality of tooth-colored restorations is a determinant factor in the esthetics and longevity of such restorations $(4,15)$. Restorations with rough surfaces enhance plaque accumulation and stain retention and may cause gingival irritation and dental caries $(3,16)$. The results of the present study indicated that all the prophylaxis treatments of giomer resulted in significant increase in surface roughness in comparison with the control group, and APD group showed a dramatic increase in surface roughness followed by pumice with brush, and pumice with rubber cup.

Stylus profilometry is an established technique for evaluation of the most commonly reported surface roughness parameter, Ra (17). AFM has several advantages for surface analysis, including higher resolution and the ability to provide $3 \mathrm{D}$ topographic images of the surface and suitability for qualitative and quantitative comparison of surface texture and roughness (18). In the current study, the surface roughness data obtained from AFM images confirmed the results obtained from profilometry and there was a strong agreement between the two techniques. As it can be observed in figure 2, the surface roughness has the same trend in both methods. The highest surface roughness values, in descending order, were observed with APD, pumice with brush, pumice with rubber cup and control group. The slight difference observed can be attributed to the small difference between the samples, higher resolution of AFM, stylus tip size limitation of the profilometry and the fact that the two variables are calculated differently; while $\mathrm{Ra}$ and Rms are both representations of roughness in terms of height measurements on the peaks and valleys, the measurements are used in a different formula. The Ra roughness is calculated along a line as in Eq. (1):

$$
R a=(1 / L) \int_{0}^{L}|Z(x)| d x
$$

where $\mathrm{L}$ is the evaluation length and $\mathrm{Z}(\mathrm{x})=$ the profile height function, on the other hand $\mathrm{Rms}$ is calculated on a surface as in Eq. (2):

$R m s=\sqrt{(1 / L) \int_{0}^{L} Z(x)^{2} d x}$

Mathematically the calculations lead to Rms being higher than Ra. It can be inferred from the definitions that a single large peak or flaw within the microscopic surface texture will positively affect (increase) the Rms value more than the Ra value. These could explain the higher values obtained from AFM, when compared to the profilometry.

Moreover, AFM analysis revealed that three prophylaxis methods significantly increased the surface area in comparison with the control group and the highest surface area, in descending order, was observed with APD, pumice with brush, pumice with rubber cup and control group. The results of the current study indicated that the more the surface roughness was, the more surface area was resulted. It seems that this is because of increased macro and micro irregularities which have been produced subsequent to different prophylaxis treatments.

Yap et al. (3) reported that in flowable, minifill and polyacid modified composites, the control group (untreated) showed the lowest surface roughness compared to the groups with different prophylaxis methods. Furthermore, previous studies have shown that the surface roughness of restorative materials significantly increases following the use of prophylactic polishing pastes, tooth brushing and sonic and ultrasonic periodontal instruments $(14,16,17)$. Giomer is a biphasic restorative material and each of the phases differs in hardness values with no uniform abrasion (19). The matrix phase in resin-based composite materials is preferentially removed during prophylaxis procedures since the abrasives in prophylactic agents are harder than the matrix. Therefore, the fillers are exposed and left unsupported. Subsequently, displacement of fillers will lead to an increased surface roughness $(3,19)$. The results of this study revealed that the use of pumice with brush resulted in significant giomer surface roughening compared to pumice with rubber cup. These results coincide with the results of studies conducted on conventional glassionomer and polyacid modified composites $(3,4)$. The higher surface roughness values in the pumice-withbrush group might be attributed to the abrasive feature of rotating brush (4). The combined effect of two-body abrasive wear due to brush bristles and slurry wear by pumice might be considered a reason for increased surface roughness (3). On the contrary, in previous studies on resin-modified glass-ionomers, highly viscous glass- 
ionomers, flowable composites and minifill composites there were no significant differences in surface roughness between groups treated by pumice with brush and those treated by pumice with rubber cup $(3,4)$. Even in resin-modified glass-ionomer and highly-viscous glassionomer materials the surface roughness values following the two above-mentioned treatments were not significantly different from those of the untreated group (4). The differences in the results might be attributed to different abrasives used in the current study and those studies $(3,4)$ since the type of abrasives and their particle size can influence the abrasive action (16). Moreover, the differences in the substrate can be considered another reason for different results. It has been reported that the hardness, the initial surface roughness, filler size, filler content and water absorption of the substrate affect wear resistance $(2,3,11,16,19)$. The specimens in the current study were stored in water for three months before polishing. Water absorption gives rise to resin matrix swelling and stress formation. Therefore, partial or complete debonding of the fillers in the surface layer can result in surface roughening (19).

In the present study, the use of APD resulted in the roughest surface in giomer samples, which confirms the results of previous studies conducted on composite resins and glass-ionomers $(3,4)$. It seems that high pressure of air and water in APD is strong enough to degrade the filler-resin bond joined together through silane. Therefore, the fillers from the superficial layer are debonded. Furthermore, the possibility of the abrasion of filler phase of resin materials by powder components of APD has been reported (3). The surface roughness of the giomer specimens treated with all prophylaxis methods was greater than $0.2 \mu \mathrm{m}$, which is a threshold value for bacterial adherence (14). Therefore, re-polishing of giomer restorations subsequent to the prophylaxis treatments tested might be necessary. In addition, according to the results of the present study, the use of pumice with rubber cup results in the least increase in surface roughness. It is suggested that this method should be preferred over the two other techniques investigated for dental prophylaxis, where giomer restorations are present. Given the results of the current study, further investigations on the surface roughness and abrasion resistance of giomer restorative materials are warranted. Within the limitations of this study it was concluded that the use of different prophylaxis methods, including pumice with rubber cup, pumice with brush and APD resulted in a significant increase in giomer surface roughness compared to untreated group. The roughest surface of giomer, in descending order, was achieved subsequent to the use of APD, pumice with brush, and pumice with rubber cup.

\section{References}

References with links to Crossref - DOI

1. López-Píriz R, Aguilar L, Giménez MJ. Management of odontogenic infection of pulpal and periodontal origin. Med Oral Patol Oral Cir Bucal. 2007;12:E154-9.

2. Agger MS, Hörsted-Bindslev P, Hovgaard O. Abrasiveness of an air-powder polishing system on root surfaces in vitro. Quintessence Int. 2001;32:407-11.

3. Yap AU, Wu SS, Chelvan S, Tan ES. Effect of hygiene maintenance procedures on surface roughness of composite restoratives. Oper Dent. 2005;30:99-104.

4. Wu SS, Yap AU, Chelvan S, Tan ES. Effect of prophylaxis regimens on surface roughness of glass ionomer cements. Oper Dent. 2005;30:180-4.

5. Shintani H, Satou J, Satou N, Hayashihara H, Inoue T. Effects of various finishing methods on staining and accumulation of Streptococcus mutans HS-6 on composite resins. Dent Mater. 1985;1:225-7. 6. Weaks LM, Lescher NB, Barnes CM, Holroyd SV. Clinical evaluation of the Prophy-Jet as an instrument for routine removal of tooth stain and plaque. J Periodontol. 1984;55:486-8.

7. Berkstein S, Reiff RL, McKinney JF, Killoy WJ. Supragingival root surface removal during maintenance procedures utilizing an air-powder abrasive system or hand scaling. An in vitro study. J Periodontol. 1987;58:327-30.

8. Petersilka GJ, Bell M, Häberlein I, Mehl A, Hickel R, Flemmig TF. In vitro evaluation of novel low abrasive air polishing powders. $J$ Clin Periodontol. 2003;30:9-13.

9. Deliperi S, Bardwell DN, Wegley C, Congiu MD. In vitro evaluation of giomers microleakage after exposure to $33 \%$ hydrogen peroxide: self-etch vs total-etch adhesives. Oper Dent. 2006;31:227-32.

10. Sunico MC, Shinkai K, Katoh Y. Two-year clinical performance of occlusal and cervical giomer restorations. Oper Dent. 2005;30:282-9.

11. McCabe JF, Rusby S. Water absorption, dimensional change and radial pressure in resin matrix dental restorative materials. Biomaterials. 2004;25:4001-7.

12. Wilson NH, Gordan VV, Brunton PA, Wilson MA, Crisp RJ, Mjör IA. Two-centre evaluation of a resin composite/ self-etching restorative system: three-year findings. J Adhes Dent. 2006;8:47-51. 13. Gordan VV, Mondragon E, Watson RE, Garvan C, Mjör IA. A clinical evaluation of a self-etching primer and a giomer restorative material: results at eight years. J Am Dent Assoc. 2007;138:621-7.

14. Lai YL, Lin YC, Chang CS, Lee SY. Effects of sonic and ultrasonic scaling on the surface roughness of tooth-colored restorative materials for cervical lesions. Oper Dent. 2007;32:273-8.

15. Mohamed-Tahir MA, Yap AU. Effects of $\mathrm{pH}$ on the surface texture of glass ionomer based/containing restorative materials. Oper Dent. 2004;29:586-91.

16. Neme AM, Wagner WC, Pink FE, Frazier KB. The effect of prophylactic polishing pastes and toothbrushing on the surface roughness of resin composite materials in vitro. Oper Dent. 2003;28:808-15.

17. Neme AL, Frazier KB, Roeder LB, Debner TL. Effect of prophylactic polishing protocols on the surface roughness of esthetic restorative materials. Oper Dent. 2002;27:50-8.

18. Fawzy AS, El-Askary FS, Amer MA. Effect of surface treatments on the tensile bond strength of repaired water-aged anterior restorative micro-fine hybrid resin composite. J Dent. 2008;36:969-76.

19. Yap AU, Mok BY. Surface finish of a new hybrid aesthetic restorative material. Oper Dent. 2002;27:161-6.

\section{Acknowledgments}

The authors extend their gratitude to the Vice Chancellor for Research at Tabriz University of Medical Sciences for their financial support. Furthermore, the authors would like to thank Dr. M Abdolrahimi (DDS), who edited the English manuscript of this article and Dr. MR Naimi-Jamal for his assistance in AFM imaging. The authors also thank Shofu Dental Corporation for supplying the giomer material. 